\title{
Altered expression of adhesion molecules and epithelial-mesenchymal transition in silica-induced rat lung carcinogenesis
}

\author{
David Blanco ${ }^{1}$, Silvestre Vicent ${ }^{1}$, Eider Elizegi ${ }^{1}$, Irene Pino ${ }^{1}$, Mario F Fraga ${ }^{3}$, \\ Manel Esteller ${ }^{3}$, Umberto Saffiotti ${ }^{2}$, Fernando Lecanda ${ }^{1, *}$ and Luis M Montuenga ${ }^{1, *}$ \\ ${ }^{1}$ Department of Histology and Pathology and Division of Oncology (Center for Applied Biomedical Research, \\ CIMA), University of Navarra, Pamplona, Spain; ${ }^{2}$ Center for Cancer Research, National Cancer Institute, \\ Bethesda, MD, USA and ${ }^{3}$ Cancer Epigenetics Laboratory, Molecular Pathology Program, Spanish National \\ Cancer Center (CNIO), Madrid, Spain
}

\begin{abstract}
Loss of the epithelial phenotype and disruption of adhesion molecules is a hallmark in the epithelialmesenchymal transition (EMT) reported in several types of cancer. Most of the studies about the relevance of adhesion and junction molecules in lung cancer have been performed using established tumors or in vitro models. The sequential molecular events leading to EMT during lung cancer progression are still not well understood. We have used a rat model for multistep lung carcinogenesis to study the status of adherens and tight junction proteins and mesenchymal markers during EMT. After silica-induced chronic inflammation, rats sequentially develop epithelial hyperplasia, preneoplastic lesions, and tumors such as adenocarcinomas and squamous cell carcinomas. In comparison with normal and hyperplastic bronchiolar epithelium and with hyperplastic alveolar type II cells, the expression levels of E-cadherin, $\alpha$-catenin and $\beta$-catenin were significantly reduced in adenomatoid preneoplastic lesions and in late tumors. The loss of E-cadherin in tumors was associated with its promoter hypermethylation. $\alpha$ - and $\beta$-catenin dysregulation lead to cytoplasmic accumulation in some carcinomas. No nuclear $\beta$-catenin localization was found at any stage of any preneoplastic or neoplastic lesion. Zonula occludens protein-1 was markedly decreased in $66 \%$ of adenocarcinomas and in $100 \%$ squamous cell carcinomas. The mesenchymal-associated proteins $\mathrm{N}$-cadherin and vimentin were analyzed as markers for EMT. N-cadherin was de novo expressed in $32 \%$ of adenocarcinomas and $33 \%$ of squamous cell carcinomas. Vimentin-positive tumor cells were found in $35 \%$ of adenocarcinomas and $88 \%$ of squamous cell carcinomas. Mesenchymal markers were absent in precursor lesions, both hyperplastic and adenomatoid. The present results show that silica-induced rat lung carcinogenesis is a good model to study EMT in vivo, and also provide in vivo evidence suggesting that the changes in cell-cell adhesion molecules are an early event in lung carcinogenesis, while EMT occurs at a later stage.
\end{abstract}

Laboratory Investigation (2004) 84, 999-1012, advance online publication, 14 June 2004; doi:10.1038/labinvest.3700129

Keywords: adhesion molecules; cadherin; catenin; tight junction; lung cancer; EMT; DNA methylation

Cell-to-cell and cell-matrix contacts, necessary for proper epithelial tissue homeostasis and function, ${ }^{1}$ are mediated by multiprotein specialized structures known as adherens and tight junctions. The basic element of adherens junctions is the cadherin/ catenin complex, required for interaction between neighboring cells. Tight junctions are formed by a

Correspondence: Dr LM Montuenga, PhD, Carcinogenesis Unit, CIMA, Department of Histology and Pathology, School of Medicine, University of Navarra, Pamplona 31080, Spain.

E-mail: lmontuenga@unav.es

*Both have contributed equally to this paper.

Received 15 January 2004; revised 24 March 2004; accepted 13 April 2004; published online 14 June 2004 complex of transmembrane proteins, including claudins and occludins, and other submembranous cytoplasmic proteins such as zonula occludens protein $1(\mathrm{ZO}-1){ }^{2}$ E-cadherin, the main cadherin expressed in adherens junctions in normal epithelia, belongs to a family of transmembrane glycoproteins with a single spanning domain that mediates calcium-dependent cell adhesion. E-cadherin represents a classical type I cadherin, and its short intracellular tail binds to cytoplasmic proteins of the catenin family, $\alpha, \beta$ and $\gamma$, forming a link with cytoskeletal actin filaments. This association with the cytoskeleton deeply influences cell behavior and motility through specific signaling molecules shuttling to the nucleus. ${ }^{3}$ In lung cancer, as in many 
other types of epithelial cancer, downregulation of E-cadherin and other adherens junctions components including $\alpha$ - and $\beta$-catenins has been reported. ${ }^{4-6}$ Recently, it has been shown that repression of the E-cadherin gene in human primary tumors is caused by aberrant hypermethylation of its promoter. ${ }^{7}$

Nonepithelial cadherins have been found to be de novo expressed in epithelial cancers in the course of an epithelial to mesenchymal transition (EMT) that has been described in epithelial carcinogenesis. ${ }^{8,9}$ EMT is a key mechanism during normal development of multicellular organisms. During this process, epithelial cells progressively acquire a reversible or irreversible mesenchymal phenotype that is essential for organogenesis. ${ }^{10}$ Morphogenetic EMT is aberrantly recapitulated during tumorigenesis in a variety of epithelial cancers including thyroid, liver, kidney, prostate, breast and lung. ${ }^{10,11}$ The common signature of this process involves disruption of normal epithelial integrity, with loss of morphological features of polarized epithelia, and partial or total gain of mesenchymal markers with progressive acquisition of a motile and invasive phenotype. $^{8}$ In addition to disrupted epithelial morphology, dysregulation of adhesion and junctional molecules and aberrant expression of $\mathrm{N}$-cadherin, EMT involves de novo expression of other mesenchymal markers such as fibronectin and vimentin in epithelial cells. N-cadherin, also a type I cadherin, is not normally expressed in bronchoalveolar epithelium and is characteristic of nerve cells, developing skeletal muscle, embryonic and mature cardiac muscle cells, pleural mesothelial cells and osteoblasts. ${ }^{12}$ It is also present in epithelial tumor cells undergoing EMT. ${ }^{8,9}$ Fibronectin, vimentin and other nonepithelial proteins have also been found abnormally expressed in epithelial tumors. ${ }^{13-15}$ Vimentin is a class III intermediate filament widely expressed during embryonic development in tissues of mesenchymal origin. ${ }^{16}$ Its aberrant expression in tumors and transformed cell lines has been correlated with increased motility, invasive behavior and poor prognosis. ${ }^{17,18}$ In lung, it is found in fibroblasts, smooth muscle, endothelial and lymphoid cells, but it is not expressed in the normal airway epithelial cells and there are no published data on its expression during lung carcinogenesis. Most of the studies about adhesion molecules and EMT in neoplasia have been performed on in vitro models, or on biopsies from fully established invasive tumors. The multistep nature of the events leading from a normal airway epithelial tissue to a tumor has been proposed..$^{19}$ Information concerning the molecular events associated with these progressive changes is still sparse and incomplete. For instance, sequential changes in the expression of adherens and tight junction molecules and mesenchymal markers have not been investigated during multistep lung cancer progression. The alteration of adherens and tight junction components and the development of EMT during multistep carcinogenesis has not been reported so far in an appropriate animal model, which could be very useful to identify and validate markers of tumor progression and invasion, and potential targets for therapeutic intervention.

In the present study, we have used the silicainduced rat model of multistep lung carcinogenesis, which mimics many features observed in human peripheral lung cancer. ${ }^{20-23}$ In this model, a single intratracheal instillation of crystalline silica dust suspended in saline leads to the appearance of pulmonary silicosis and of proliferative epithelial lesions with progressive severity, from alveolar and bronchiolar epithelial hyperplasias to preneoplastic adenomatoid lesions and to adenocarcinomas and squamous cell carcinomas (See Materials and methods, Animal model). Adenocarcinomas were classified into fibrotic or nonfibrotic depending on the degree of fibrosis present. In squamous cell carcinomas, two clearly distinguishable areas were found namely: (a) well differentiated tumors with a central area filled with keratin and (b) smaller irregular solid cords of cells infiltrating into the adjacent connective tissue, in some cases in direct contact with the differentiated areas. We analyzed changes in the expression of E-cadherin/ $\alpha$-catenin/ $\beta$-catenin proteins, ZO-1 tight junction protein, and the mesenchymal markers $\mathrm{N}$-cadherin and vimentin during lung cancer progression. We observed markedly reduced protein expression of E-cadherin, $\alpha$-catenin and $\beta$-catenin in tumors and preneoplastic lesions. By contrast, reduction/absence of ZO-1 and neoexpression of $\mathrm{N}$-cadherin and vimentin were only observed in tumors.

\section{Materials and methods}

\section{Animal Model}

Fisher F344/NCr female rats from Harlan UK Limited (Oxon, UK) were employed. The crystalline silica sample was $99 \%$ pure $\alpha$-quartz (Min-U-Sil 5, US Silica Co., Berkeley Springs, WV, USA), with particle size less than $5 \mu \mathrm{m} \cdot{ }^{20,22}$ The silica sample was suspended in sterile neutral buffered saline and briefly sonicated to provide full dispersion. Rats at 8 weeks of age were anesthetized with a mixture of oxygen and isofluorane, and placed on their backs on a metal board slanted at a $60^{\circ}$ angle with the mouth kept open, and in this position, at the end of an expiration, they received a single intratracheal instillation of $16 \mathrm{mg}$ quartz in $0.3 \mathrm{ml}$ saline through a 7-cm-long, 19-gauge stainless steel blunt cannula, bent at a $140^{\circ}$ angle near the top, connected to a syringe..$^{20}$ A total of 36 rats were instilled with $16 \mathrm{mg}$ quartz and the lungs obtained at months $1(n=5), 4$ $(n=5), 11(n=2), 17(n=7)$ and $21(n=17)$ after instillation. Several lesions were studied at these time periods: hyperplasias of type II pneumocytes $(n=27)$ beginning from month 1 , hyperplasia of 
bronchiolar epithelial cells $(n=19)$ from month 4 , preneoplastic adenomatoid lesions $(n=28)$ from month 8 , and adenocarcinomas $(n=28)$ and squamous cell carcinomas $(n=9)$ from month 11 . Hyperplastic and preneoplastic lesions were observed not only at the time of appearance but also in late stages. Tissues from control rats $(n=8)$, instilled with $0.3 \mathrm{ml}$ saline without quartz, were obtained at months $6(n=4)$ and $12(n=4)$. The rats were housed in specific pathogen-free conditions with access to food and water ad libitum. Procedures were carried out in strict compliance of the EU and NIH relevant guidelines for use of laboratory animals. The rats were killed by exsanguination under anesthesia with Ketolar $^{\mathbb{B}}$ (Parke-Davis, Madrid, Spain) and Rompún ${ }^{\circledR}$ (Bayer AG, Leverkusen, Germany). The trachea was exposed by dissection and ligated during maximal inspiration. The larynx, trachea, bronchi, lungs, lymph nodes and heart were removed 'en bloc' and fixed by immersion in $4 \%$ formaldehyde in a $300 \mathrm{mOsm}$ phosphate buffer for $24 \mathrm{~h}$. Lung lobes were sectioned along their main bronchial axis, embedded in paraffin, and sectioned at $4-\mu \mathrm{m}$ thickness. Paraffin-embedded lungs from treated and control rats were used. Additional specimens $(n=24)$ were obtained from previous analogous experiments carried out at the National Cancer Institute (NCI), Bethesda, MD. ${ }^{20,22}$

A subset of the late tumor was isolated from the fresh unfixed lung and split into two parts. One of the two halves was rapidly snap frozen in liquid nitrogen and kept at $-80^{\circ} \mathrm{C}$ for DNA extraction. The other half of the tumor was processed for histological analysis. Normal lung from control rats was processed in the same way.

\section{Immunohistochemistry}

\section{Antibodies}

For immunohistochemical analysis, the following antibodies (Abs) tested for cross reactivity for human and rat were used: anti-E-cadherin (C36) (class, IgG2a mouse monoclonal) (BD Transduction Laboratories, San Diego, CA, USA); anti- $\beta$-catenin (E-5) (class, IgG1 mouse monoclonal; epitope, residues $680-781$ of $\beta$-catenin, human origin) (Santa Cruz Biotechnology, Santa Cruz, CA, USA); anti- $\beta$-catenin (C14) (class, IgG1 mouse monoclonal) (BD Transduction Laboratories, San Diego, CA, USA); anti- $\alpha$-catenin (class, IgG1 mouse monoclonal) (Calbiochem, EMD Bioscience, Darmstadt, Germany); anti-N-cadherin (3B9) (class, IgG1 mouse monoclonal) (Zymed Laboratories, San Francisco, CA, USA); anti-ZO-1 (class, Ig rabbit polyclonal; epitope, residues 463-1109 of ZO-1, human origin) (Zymed Laboratories, San Francisco, CA, USA); anti-vimentin (V9) (class, IgG1 mouse monoclonal) (Dako, Barcelona, Spain); anti-cytokeratins (MNF-116) (class, IgG1 mouse monoclonal) (Dako, Barcelona, Spain).
Methods

For the immunohistochemical technique, the indirect avidin-biotin-peroxidase method (Dako, Barcelona, Spain) was employed in the analysis of E-cadherin, $\beta$-catenin, $\alpha$-catenin and cytokeratins. The EnVision ${ }^{\circledR}$ (K4001 and K4007; Dako) signal enhancement system was used in the analysis of $\mathrm{N}$-cadherin, ZO-1 and vimentin. Slides were deparaffined and incubated for $10 \mathrm{~min}$ with $3 \% \mathrm{H}_{2} \mathrm{O}_{2}$ in water to quench the endogenous peroxidase activity. Heat-mediated antigen retrieval was used for antibodies against $\mathrm{E}$-cadherin, $\beta$-catenin, $\alpha$-catenin, $\mathrm{N}$-cadherin and vimentin (microwave treatment, $15 \mathrm{~min}$ at $750 \mathrm{~W}$ and $15 \mathrm{~min}$ at $375 \mathrm{~W}$ ) and an enzymatic antigen retrieval method was employed for antibodies against cytokeratins $\left(7 \mathrm{~min}\right.$ at $\left.37^{\circ} \mathrm{C}\right)$ (protease type XIV, Sigma-Aldrich, Madrid, Spain), and ZO-1 (12 min at $37^{\circ} \mathrm{C}$ ) (protease type XXIV, Sigma-Aldrich, Madrid, Spain). Tissues were incubated with $5 \%$ normal rabbit serum in Tris-buffered saline (TBS) (Tris-HCl $0.05 \mathrm{M}, \quad 0.5 \mathrm{M} \mathrm{NaCl}$, $\mathrm{PH}=7.36$ ) for $30 \mathrm{~min}$ at room temperature. After blotting the excess serum, the sections were incubated with the primary antibody diluted in TBS at $4{ }^{\circ} \mathrm{C}$ overnight. Dilutions were: $1: 50$ for antiE-cadherin, $\alpha$-catenin and ZO-1; 1:100 for anti$\beta$-catenin (E-5); 1:200 for anti- $\beta$-catenin (C-14), $\mathrm{N}$-cadherin, cytokeratins and vimentin. Tissues were washed in TBS and incubated with the appropriate secondary antibody. For the indirect avidin-biotin-peroxidase method, biotinylated rabbit anti-mouse Ig antiserum was added at a 1:200 dilution for $30 \mathrm{~min}$ at room temperature, and after washing, the slides were incubated $30 \mathrm{~min}$ at room temperature with the avidin-biotin complex at a 1:100 dilution. In the EnVision ${ }^{\mathbb{B}}$ signal enhancement system, the secondary monoclonal or polyclonal complex was used for $30 \mathrm{~min}$ at room temperature. After washing the slides in TBS, development of peroxidase with diaminobenzidine and $\mathrm{H}_{2} \mathrm{O}_{2}$ was performed. After rinsing in water, the sections were counterstained with Harris hematoxylin, dehydrated and mounted with distyrene, plasticizer, xylene (DPX) mounting medium (BDH, Poole, England).

\section{Evaluation of immunostaining}

Several histological types were analyzed in each section, namely normal cells: normal bronchiole (B), normal type II pneumocytes (TII Pn); hyperplastic lesions: bronchiolar hyperplasia $(\mathrm{BH})$ and type II pneumocyte hyperplasia (TII $\mathrm{PnH}$ ); preneoplastic lesions: adenomatoid lesions (AL); and tumors: adenocarcinoma (AC) and squamous cell carcinoma (SCC).

To assess E-cadherin, $\beta$-catenin, $\alpha$-catenin and ZO1 status quantitative evaluation of immunostained sections was performed according to previously published methods. ${ }^{24}$ Normal bronchioles present in normal tissues or adjacent to the lesion were used as internal control of staining. Staining intensity 
was graded as normal (value 3 , intensity $>75 \%$ of the normal bronchiolar staining), low (value 2, intensity $50-75 \%$ of the normal bronchiolar staining), very low (value 1 , intensity $25-50 \%$ of the normal bronchiolar staining) and no staining (value 0 , intensity $<25 \%$ of the normal bronchiolar staining). The percentages of area were also assigned from 100 to $0 \%$. Multiplication of the intensity score and the percentage area gave a final multiplication value from 300 to 0 . The multiplication values were grouped into four immunoreactive scores defined as 'normal' (multiplication values 300-201), 'low' (multiplication values 200-101), 'very low' (multiplication values 100-1) and 'negative' (multiplication value $=0$ ). Scoring was carried out independently by two independent observers with an initial scoring agreement higher than 90\%. Consensus score was annotated for the discrepant sections after joint review of these particular cases.

To analyze $\mathrm{N}$-cadherin and vimentin status, sections from each block were immunostained for N-cadherin and vimentin. Normal bronchiolar epithelial cells present in normal tissues or adjacent to the lesion were used as internal control of negative staining. Mesenchymal cells such as fibroblasts were used as internal controls. Number and size of stained cell foci were recorded for each section.

\section{Statistical analysis}

Statistical analysis was performed with the SPSS 9.0 software (SPSS Inc., Chicago, IL, USA). A KruskalWallis test was applied to the study of E-cadherin, $\beta$-catenin and $\alpha$-catenin. In the cases for which significant statistical variations were present, comparison of preneoplastic and neoplastic epithelial lesions with the corresponding normal bronchioles and hyperproliferative lesions was calculated using a Mann-Whitney $U$-test with the Bonferroni adjustment. A Fisher exact test was applied to the study of vimentin in fibrotic and nonfibrotic adenocarcinomas.

\section{Controls}

Negative controls: Mouse IgG1 and IgG2a (Dako, Barcelona, Spain) with specificity directed towards Aspergillus niger glucose oxidase were used as negative controls for immunohistochemical techniques. Nuclear staining validation for $E-5$ and $C-14$ $\beta$-catenin antibodies: Human colon carcinoma sections were used to validate the nuclear staining of $\beta$-catenin. Both of them stained the nuclei in the control tumoral cells (data not shown).

\section{Quantification of Global DNA Methylation}

Genomic DNA was extracted from the frozen tumors by conventional methods. The 5-methylcytosine DNA content of rat lung tumors (adenocarcinomas, $n=5$; and squamous cell carcinomas, $n=4$ ) and control rat lungs $(n=6)$ was determined by highperformance Capillary Electrophoresis (HPCE) as previously described. ${ }^{25,26}$ Briefly, genomic DNA (3-5 $\mu \mathrm{g})$ was obtained from the different tissues and DNA hydrolysis was carried out with $1.25 \mu \mathrm{l}$ $(200 \mathrm{U} / \mathrm{ml})$ of nuclease P1 for $16 \mathrm{~h}$ at $37^{\circ} \mathrm{C}$. Subsequently, alkaline phosphatase was added and mixtures were incubated for an additional $2 \mathrm{~h}$ at $37^{\circ} \mathrm{C}$. Hydrolyzed samples were injected under pressure (0.3 p.s.i.) for $3 \mathrm{~s}$ into an uncoated fused-silica capillary in a $\mathrm{CE}$ system $\left(\mathrm{P} / \mathrm{AC}^{\mathrm{TM}} \mathrm{MDQ}\right.$, BeckmanCoulter). Quantification of the relative methylation of each DNA sample was determined as the percentage of $\mathrm{mC}$ of total cytosines: $\mathrm{mC}$ peak area $\times 100 /(C$ peak area $+\mathrm{mC}$ peak area $)$. All samples were analysed in duplicate and three analytical measurements were made per replicate.

\section{Bisulfite Genomic Analysis of the CDH1 CpG Island}

DNA samples of five adenocarcinomas (Tumors 3, 4, 6,8 and 9), four squamous cell carcinomas (Tumors 1, 2, 5, and 7) and six normal lungs (Normal 1-6) were treated with sodium bisulfite as previously described $^{27}$ and primers (Figure 3c) spanning the $\mathrm{CpG}$ island of the rat E-cadherin ( $C D H 1)$ promoter were used for bisulfite genomic sequencing. ${ }^{28}$ At least six individual clones were sequenced for each sample. A single $\mathrm{CpG}$ was considered to be methylated (black dot) when more than half of the clones retained an unmodified cytosine at that position.

\section{Results}

First, we analyzed the expression of proteins involved in cell-to-cell adhesion: E-cadherin, $\beta$-catenin and $\alpha$-catenin. Strong membranous staining for E-cadherin was found both in normal bronchiolar epithelial cells (Figure 1a) and hyperplastic bronchiolar epithelium (Figure 1b), with no statistical difference in the staining score. While normal isolated type II pneumocytes showed very low levels of E-cadherin (Figure 1c), hyperplastic type II pneumocytes areas $(n=27)$ expressed significantly higher amount of the protein, which was also localized at the plasma membrane (Figure 1d). In adenomatoid lesions $(n=28)$ (Figure 1e), E-cadherin immunoreactivity was considerably reduced as compared to normal bronchiole and hyperplastic lesions (Figure 2), $P<0.001$ compared to normal bronchiole, bronchiolar hyperplasia and type II pneumocyte hyperplasia. At the final time point of the experiment (months 17-21) two different tumor types were found: adenocarcinomas $(n=28)$, which were more frequent, and squamous cell carcinomas $(n=9)$. E-cadherin was significantly reduced in both types of tumors (Figure 1f,g) as compared to normal and hyperplastic bronchiolar epithelium (Figure 2, $P<0.001$ for both comparisons). 



Figure 1 Immunohistochemistry of E-cadherin during silica-induced rat lung carcinogenesis. Expression in normal bronchiolar (a) and alveolar (c) epithelium and in hyperplastic bronchiolar (b) and alveolar (d) epithelium. Very low expression in normal type II pneumocytes (c) compared to hyperplastic type II pneumocytes (d) Inset: detail of the type II pneumocyte hyperplasia shown in (d) (e-g) E-cadherin expression was decreased (arrowhead) in adenomatoid lesions (e), adenocarcinoma (f) and squamous cell carcinoma (g) compared to normal (a) and hyperplastic (b) bronchiolar epithelium. Nuclear counterstain in all figures is with Harris hematoxylin. Original magnifications: $\times 120(\mathbf{b}, \mathbf{d}-\mathbf{g}) ; \times 300$ (a,c,d inset). BrE: Bronchiolar epithelium. 


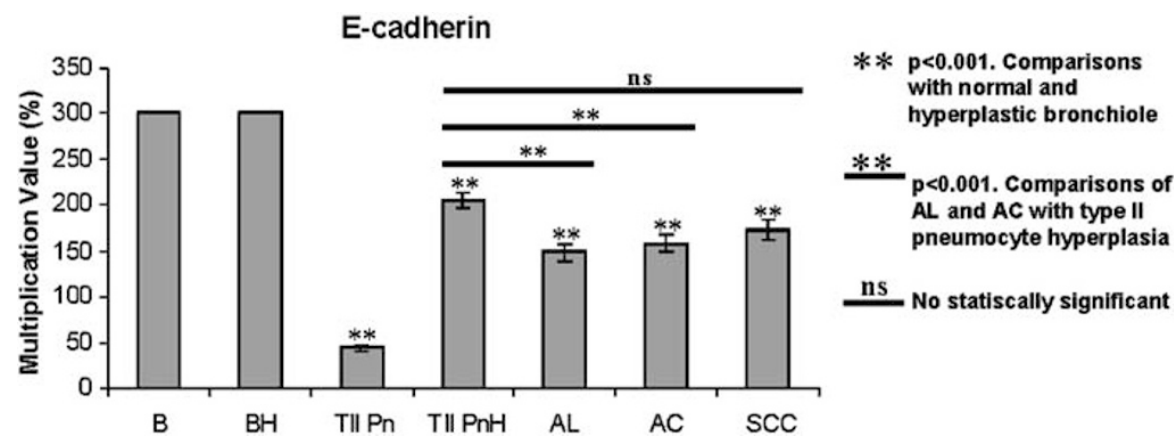

Figure 2 Statistical analysis of the expression of E-cadherin. Semiquantitative analysis of the expression of E-cadherin in the normal epithelia and in the different types of lesions. Normal bronchiole adjacent to the lesion was used as internal positive control of staining efficiency and as an internal standard for quantitation. Normal cells: normal bronchiole (B), normal type II pneumocytes (TII Pn); hyperplastic lesions: bronchiolar hyperplasia (BH) and type II pneumocyte hyperplasia (TII PnH); preneoplastic lesions: adenomatoid lesions (AL); and tumors: adenocarcinoma (AC) and squamous cell carcinoma (SCC). A Kruskal-Wallis test was applied and the post hoc pairwise comparisons were calculated using a Mann-Whitney $U$-test with the Bonferroni adjustment.

Moreover, E-cadherin was significantly reduced in adenocarcinomas as compared to hyperplastic type II pneumocytes (Figure 2, $P<0.001$ ). There was also a reduction in E-cadherin expression in squamous cell carcinomas as compared to hyperplastic type II pneumocytes although it was not statistically significant (Figure 2, NS).

The potential epigenetic regulation of gene expression in this model of multistage lung carcinogenesis was first explored through the quantification of the global DNA methylation levels of adenocarcinomas $(n=5)$ and squamous cell carcinomas $(n=4)$ vs control lung tissues $(n=6)$ (Figure 3a). As it was previously reported for human breast and colorectal primary tumors, ${ }^{26}$ silica-induced carcinomas showed a clear global genomic hypomethylation, with an average loss of $25 \%$ of 5 -methylcytosine DNA content compared to the normal tissues. Next, we determined the role of promoter hypermethylation in the transcriptional repression of the Ecadherin gene in nine tumors and six saline instilled control lungs (Figure 3d). We observed a CpG island hypermethylation pattern in six of nine tumors analyzed. Furthermore, we were able to study the correlation between the E-cadherin protein expression and the promoter hypermethylation status in Tumors 1-3. In these samples, direct bisulfite sequencing analyses of the promoter region of E-cadherin revealed a strong direct association between E-cadherin promoter hypermethylation (Figure 3d) and marked loss of E-cadherin protein expression (Figure 3b) in Tumor 2 (squamous cell carcinoma, Figure 1g) and Tumor 3 (adenocarcinoma, Figure 7a). However, Tumor 1 (squamous cell carcinoma) showed normal E-cadherin expression (Figure 3b) and its promoter region remained unmethylated (Figure 3d).

The $\beta$-catenin expression profile was similar to Ecadherin in normal tissues and in all lesions studied (Figure 4a-h, and Figure 5). Strong membrane staining of $\beta$-catenin was found in normal (Figure $4 a)$ and hyperplastic $(n=19)$ (Figure $4 b)$ bronchiolar epithelium, and those levels were not statistically different. Hyperplastic type II pneumocytes lesions $(n=27)$ (Figure $4 \mathrm{~d})$ expressed significantly higher levels of $\beta$-catenin compared to normal type II pneumocytes (Figure 4c). Expression of $\beta$-catenin was reduced in adenomatoid lesions $(n=28)$ (Figure 4e) compared to normal bronchiole and hyperplastic lesions (Figure 5, $P<0.001$ compared to normal bronchiole, bronchiolar hyperplasia and type II pneumocyte hyperplasia). In adenocarcinomas $(n=28)$ and squamous cell carcinomas $(n=9)$, $\beta$-catenin was significantly reduced (Figure 4f,g) compared to normal bronchiole and hyperplastic lesions (Figure 5, $P<0.001$ compared to normal bronchiole, bronchiolar hyperplasia and type II pneumocyte hyperplasia). While adenomatoid lesions showed a membranous $\beta$-catenin staining pattern, a noticeable cytoplasmic localization of this protein was found in some of the tumors (Figure 4h). Adenocarcinomas and squamous cell carcinomas showed an exclusively granular cytoplasmic $\beta$-catenin pattern in $15 \%$ of the tumors, whereas in $67 \%$ of the tumors, $\beta$-catenin was only localized at the adherens junction complex of the plasma membrane. The remaining tumors $(18 \%)$ showed both cytoplasmic and membrane localization. We did not find nuclear localization, under our experimental conditions, in any of the tumors studied, including those with strong cytoplasmic $\beta$-catenin expression.

$\alpha$-catenin expression was always similar to Ecadherin and $\beta$-catenin in normal tissues and the lesions studied (data not shown). The mislocalization pattern of $\alpha$-catenin and $\beta$-catenin was found simultaneously in the same cells when serial sections of the tumors were analyzed.

We next studied ZO-1, a membrane-associated component of tight junctions. Normal cells showed submembranous localization at the apical region in a button like appearance, whereas basal epithelial cells showed no expression (Figure 6a, inset). Both hyperplastic bronchiolar and alveolar epithelial 
a

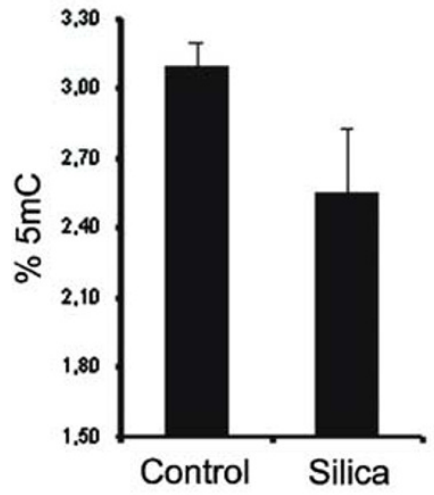

b

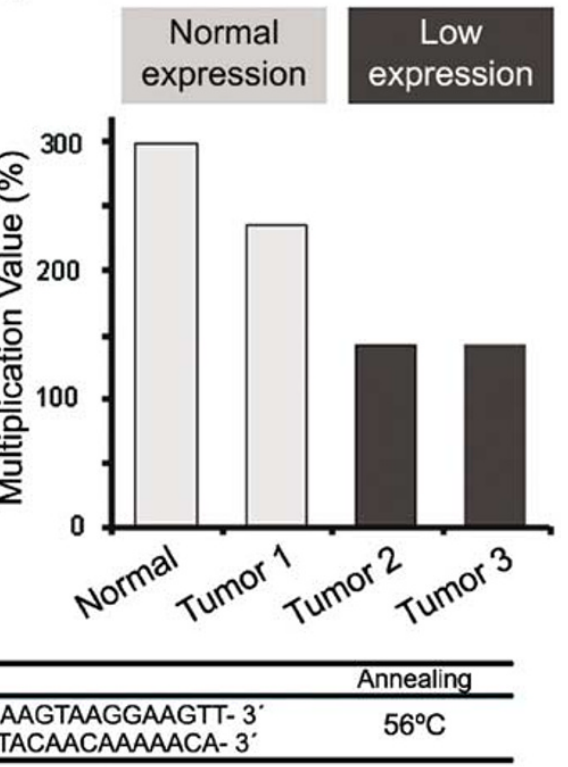

d



Figure 3 DNA methylation analysis in the normal epithelia and in tumors. (a) HPCE quantification of the global DNA methylation levels in normal lung $(n=6)$ and in silica-induced adenocarcinomas $(n=5)$ and squamous cell carcinomas $(n=4)$. Results are expressed as mean + s.d. (b) Multiplication values of the immunohistochemical signal as a measurement of the expression levels of E-cadherin in normal and Tumor 1 (squamous cell carcinoma), Tumor 2 (squamous cell carcinoma) and Tumor 3 (adenocarcinoma) samples. Light gray: normal E-cadherin protein expression; dark gray: low E-cadherin protein expression. (c) Bisulfite sequencing primers for the rat version of the E-cadherin (CDH1) promoter. (d) Bisulfite genomic sequencing of the rat E-cadherin promoter in five adenocarcinomas (Tumors 3 , 4, 6, 8 and 9), four squamous cell carcinomas (Tumors 1, 2, 5, and 7) and six normal lungs (Normal 1-6). At least six individual clones were sequenced for each sample. The localization of the CpG island obtained with the NEWCPGREPORT software (EMBOSS; http:// mammoth.bii.a-star.edu.sg/emboss/index.html) is represented with a horizontal bar. The vertical bars represent the distribution of the CpGs at the E-cadherin CpG islands and the vertical arrow indicated the transcriptional start point. Black dots indicate methylated CpGs and white dots unmethylated. The position of the bisulfite sequencing primers used is represented with white horizontal arrows. 
1006


Figure 4 Immunohistochemistry of $\beta$-catenin during silica-induced rat lung carcinogenesis. Expression in normal bronchiolar (a) and alveolar (c) epithelium and in hyperplastic bronchiolar (b) and alveolar (d) epithelium. Very low expression was found in normal type II pneumocytes (c, inset) compared to hyperplastic type II pneumocytes (d, inset). (e-g) $\beta$-catenin expression was decreased (arrowhead) in adenomatoid lesions (e), adenocarcinoma (f) and squamous cell carcinoma (g) compared to normal bronchiolar epithelium (a) and hyperplastic lesions (b, d). (h) $\beta$-catenin dysregulation leads to an accumulation in the cytoplasm (arrow) but not into the nuclei in adenocarcinoma and squamous cell carcinoma. Nuclear counterstain in all figures is with Harris hematoxylin. Original magnifications: $\times 120(\mathbf{b}-\mathbf{h}) ; \times 300(\mathbf{a}$, inset $\mathbf{c}$, inset d, inset h). BrE: Bronchiolar epithelium.

cells showed similar levels of ZO-1. Loss of expression of ZO-1 was clearly observed in neoplastic lesions compared to normal and hyperplastic bronchiolar epithelium. In adenocarcinomas, 33\% expressed low ZO-1 levels and 66\% showed very low levels (Figure 6a). Every squamous cell 


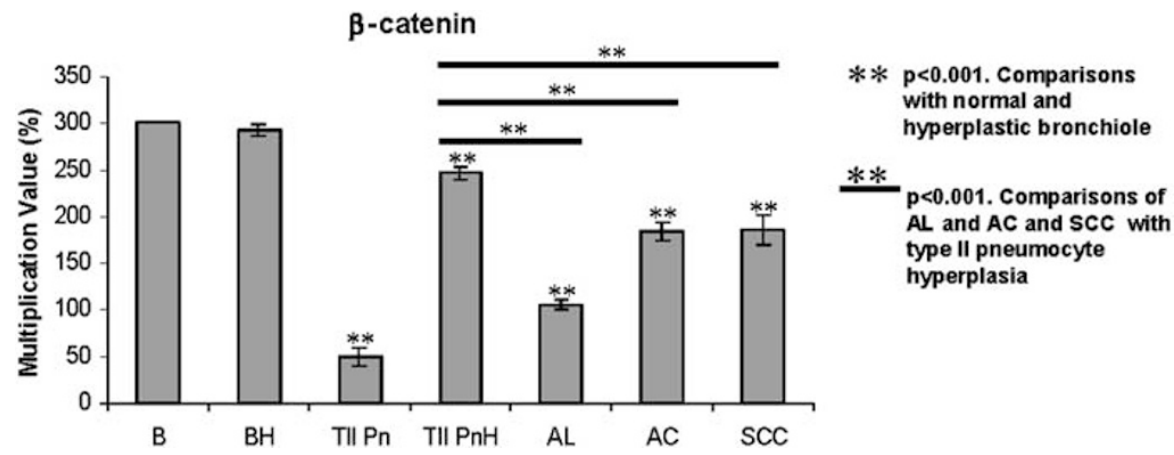

Figure 5 Statistical analysis of the expression of $\beta$-catenin. Semiquantitative analysis of the expression of $\beta$-catenin in the normal epithelia and in the different types of lesions. Normal bronchiole adjacent to the lesion was used as internal positive control of staining efficiency and as an internal standard for quantitation. Normal cells: normal bronchiole (B), normal type II pneumocytes (TII Pn); hyperplastic lesions: bronchiolar hyperplasia (BH) and type II pneumocyte hyperplasia (TII PnH); preneoplastic lesions: adenomatoid lesions (AL); and tumors: adenocarcinoma (AC) and squamous cell carcinoma (SCC). A Kruskal-Wallis test was applied and the post hoc pairwise comparisons were calculated using a Mann-Whitney $U$-test with the Bonferroni adjustment.


Figure 6 Immunohistochemistry of ZO-1 and N-cadherin in silica-induced rat lung carcinogenesis. (a) Loss of expression of ZO-1 (arrow) in adenocarcinoma compared to normal bronchiole (inset). (b) De novo expression of N-cadherin (arrowhead) in adenocarcinoma. Counterstaining by Harris hematoxylin. Original magnifications: $\times 60(\mathbf{a}) ; \times 120(\mathbf{b}) ; \times 300$ (inset a)

carcinoma studied expressed very low levels of ZO-1 expression (figure not shown).

We next studied the EMT using N-cadherin and vimentin as mesenchymal phenotypic markers (Figures $6 \mathrm{~b}$ and 7). We found N-cadherin-expressing cells in a patchy appearance in nine of 28 adenocarcinomas (Figure 6b) and in three of nine squamous cell carcinomas. We did not find any correlation between $\mathrm{N}$-cadherin expression and the degree of fibrosis in adenocarcinomas. In the squamous cell carcinoma where $\mathrm{N}$-cadherin expression was found, it was only present in the cords of infiltrating cells but not in the fully squamous differentiated area.

Vimentin was not expressed either in hyperplastic bronchioloalveolar epithelium or in adenomatoid lesions. Aberrant expression was only found in foci of cells present in 11 of 28 adenocarcinomas studied. The vimentin-positive foci were more commonly observed in fibrotic adenocarcinomas (7/8) than in the nonfibrotic adenocarcinomas (4/ $20)$, and this difference was statistically significant $(P<0.01$, Fisher's exact test).

To confirm the epithelial phenotype of the tumor cells that expressed mesenchymal markers, we used antibodies against epithelial markers like cytokeratins and E-cadherin. Coexpression of epithelial and mesenchymal markers was analyzed by immunostaining serial and face-reverse sections of the tumoral lesions with the relevant markers. Figures $7 \mathrm{a}-\mathrm{b}$ and $\mathrm{c}-\mathrm{e}$ are examples of the patterns of coexpression of epithelial and mesenchymal markers observed. We found that E-cadherin and cytokeratins co-expressed with vimentin in $100 \%$ of tumoral positive foci demonstrating that the epithelial phenotype of the tumoral lesions is preserved even when traits of mesenchymal phenotype are found (Figure $7 \mathrm{a}-\mathrm{b}, \mathrm{c}-\mathrm{d}$ ). Also, N-cadherin 

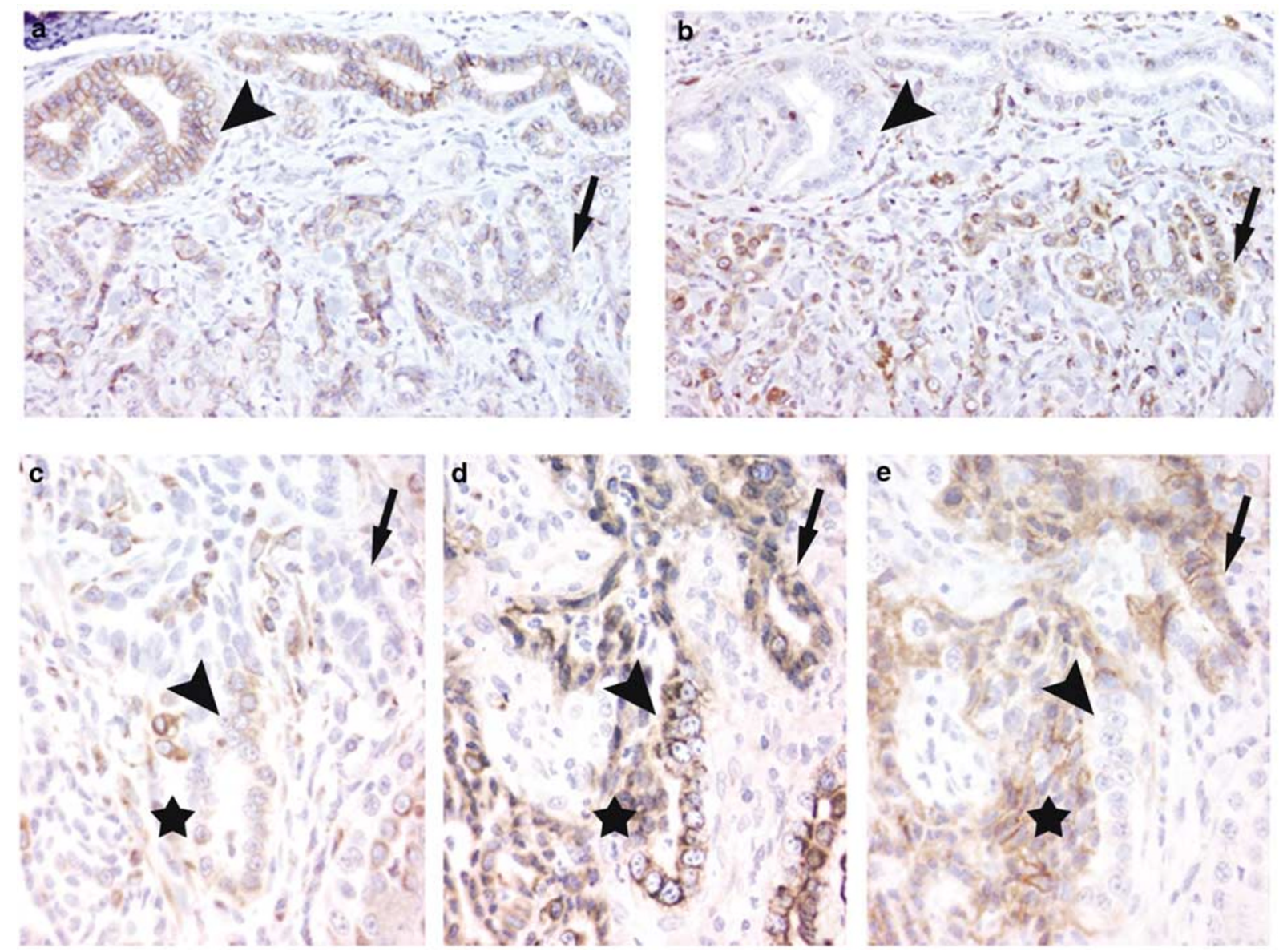

Figure 7 EMT in rat lung carcinogenesis. (a,b) Coexpression of E-cadherin (a) with vimentin (b) in EMT areas (arrow) but not in areas of well-differentiated adenocarcinoma (arrowhead), where only E-cadherin expression is observed. (c-e) Immunohistochemical study of vimentin expression (c), cytokeratins (d) and N-cadherin (e) in serial sections of adenocarcinoma. Several patterns of coexpression are observed in these images: arrowhead points to areas of simultaneous expression of vimentin and cytokeratins but not of N-cadherin; arrow shows concomitant expression of cytokeratins and N-cadherin but not of vimentin; star indicates simultaneous expression of vimentin, cytokeratins and N-cadherin. Counterstaining by Harris hematoxylin. Original magnifications: $\times 60(\mathbf{a}, \mathbf{b}) ; \times 120(\mathbf{c}-\mathbf{e})$.

co-expressed with both cytokeratin and vimentin in some tumoral areas (Figure 7e). In the squamous cell carcinomas, vimentin-expressing foci were observed in seven of nine tumors studied. Again, cytokeratins and E-cadherin coexpressed with vimentin in these tumors. As shown with $\mathrm{N}$-cadherin, vimentin expression was observed mostly in the irregular cords infiltrating the surrounding connective tissue of the squamous cell carcinoma (Figure not shown).

\section{Discussion}

In this study we have shown the alteration of adhesion molecules and the EMT in a rat model of lung carcinogenesis. In this model, we have found a progressive loss of adherens junctions components such as E-cadherin and $\alpha$ - and $\beta$-catenins, and perturbation of epithelial polarity substantiated by loss of tight junction protein ZO-1. Acquisition of the mesenchymal phenotype was evident by coexpression of vimentin and cytokeratins and de novo expression of $\mathrm{N}$-cadherin and vimentin in epithelial tumoral cells.

The present model, representing multistep lung carcinogenesis, ${ }^{20-22}$ offers several advantages over genetically modified models, such as the slow development of lesions with a progressive transition from normal to preneoplastic and to well-characterized tumors, including adenocarcinomas and squamous cell carcinomas. Another relevant feature of this model is the intimate link between chronic inflammation and carcinogenesis, similar to human lung cancer. ${ }^{29-31}$ The heterotypic interactions taking place between epithelial and immune cells have been shown to play an important role in tumor initiation, maintenance and development. ${ }^{32}$

Another interesting finding in this model was the appearance of the EMT, a common signature in many types of cancer. Although the spatiotemporal development of EMT in human tumors is not completely characterized in vivo, several reports suggest that EMT represents a potential mechanism of carcinoma progression. We have found evidence 
of EMT only in tumors but not in preneoplastic lesions. Thus, this process represents a late stage after the decrease of cell adhesion components, Ecadherin/catenin complex, which starts in preneoplastic lesions. In human carcinogenesis, only fully established lung carcinomas have been studied for E-cadherin/catenin complex alterations. ${ }^{4,33-35}$ In the present model, we have been able to study these molecules in early hyperplastic and preneoplastic lesions of bronchiolar and alveolar type II cells. We observed that $\alpha$ - and $\beta$-catenin appeared in a granular cytoplasmic pattern in tumors. Our data are in partial agreement with the aberrant expression and localization of $\beta$-catenin that has been reported in a variety of human cancers including colon, ${ }^{36}$ liver, ${ }^{37}$ stomach, ${ }^{38}$ pancreas, ${ }^{39}$ bone, ${ }^{40}$ and thyroid. ${ }^{41}$ Reports that have studied the expression of $\beta$-catenin in human lung cancer remain controversial. Some authors, in agreement with the present observations, reported restricted cytoplasmic localization and never found $\beta$-catenin nuclear translocation, ${ }^{34,42-45}$ whereas some other studies show cytoplasmic and nuclear localization of $\beta$-catenin. ${ }^{33,46-48}$ Although we did not detect $\beta$-catenin in the nucleus, we cannot rule out the possibility that $\beta$-catenin accumulation in the cytoplasm could lead to the translocation of undetectable levels of the protein to the nucleus, which could lead to the activation of target genes.

Several mechanisms have been reported to contribute to the dowregulation of key adhesion proteins. These include decrease or lack of E-cadherin/catenin expression by mutations and deletions in the genes encoding E-cadherin or catenin, promoter hypermethylation of E-cadherin, or post-translational modifications. , $^{4,45,49-51}$ To look into the specific possible causes of the downregulation of E-cadherin, we studied the DNA methylation status of its promoter region in tumors with reduced expression, since aberrant hypermethylation was demonstrated to be one of the most important mechanisms of inactivation of this adhesion protein in human ${ }^{7}$ and mouse ${ }^{52}$ cancer. Our results revealed that the promoter hypermethylation of E-cadherin may be an important mechanism for its repression in our rat model. However, we cannot discard other potential mechanisms implicated in E-cadherin repression. For instance, downregulation of E-cadherin during EMT in several human cancers could also be mediated by Slug and Snail transcriptional repressors. ${ }^{53-55}$

In addition to adherens junctions, integrity of cell-cell contacts depends also on tight junction proteins. ZO-1, the mammalian homologue of dlg, a tumor suppressor gene in Drosophila, is a component of tight junctions and can interact with adherens junctions such as $\alpha$-catenin and other adaptor proteins. ZO-1 is related to EMT, ${ }^{56}$ and has been associated with proliferation ${ }^{56,57}$ and tumor formation. ${ }^{56,58}$ A truncated ZO-1 protein containing only the Postsynaptic density/Disc large/Zona occludens-1 (PDZ) domains induced dramatic EMT in canine kidney cells. ${ }^{56}$ We observed a marked reduction of ZO-1 in tumors compared to normal epithelium, revealing an evident loss of epithelial polarity in tumors. ZO-1 has been shown to interact with a variety of transmembrane proteins including occludins, cingulin, adherens junction components, and gap junction proteins. ${ }^{56,59-61}$ This close interaction may explain a hypothetical simultaneous perturbation of adherens and tight junctions as a consequence of the same signalling event; however, when studying serial sections, we did not observe a parallel decrease of E-cadherin and reduction/ absence of ZO-1. Altered integrity of these proteins could induce aberrant signals leading to uncontrolled proliferation. ${ }^{57}$

Neoexpression of mesenchymal markers represents the hallmark of EMT. Both vimentin and Ncadherin, which are not normally expressed in epithelial cells, coexpressed with cytokeratins in neoplastic cells of tumors present in the silicainduced lung cancer rat model. De novo expression of $\mathrm{N}$-cadherin has also been shown in other human cancers and its expression was correlated with acquisition of a more invasive phenotype. For instance, a switch towards $\mathrm{N}$-cadherin expression was reported in human prostate cancer and its expression in prostate cell lines correlates with a more aggressive invasive phenotype. ${ }^{9}$ Inhibition of $\mathrm{N}$-cadherin expression in a squamous carcinomaderived cell line expressing N-cadherin-induced epithelial morphology and E-cadherin expression. ${ }^{62}$ On the other hand, induced expression of $\mathrm{N}$ cadherin in human breast cancer cells resulted in increased motility and invasion. ${ }^{63}$ Similar results were found in breast cancer cell lines where expression of $\mathrm{N}$-cadherin correlated with motility, invasion and metastasis even in the presence of $\mathrm{E}$ cadherin expression. ${ }^{64}$ We found $\mathrm{N}$-cadherin expression restricted to isolated conspicuous patchy areas within tumoral masses in adenocarcinomas and squamous carcinomas. The available in vitro data on $\mathrm{N}$-cadherin overexpression suggest that the $\mathrm{N}$ cadherin-positive cells found in our model may have a more aggressive phenotype than the rest of neoplastic cells. N-cadherin has been also proposed as a factor that promotes interaction with stromal cells. ${ }^{64}$ Indeed, N-cadherin was expressed by adenocarcinomas and by the cords of infiltrating tumoral cells in squamous cell carcinomas. This finding is consistent with its potential role mediating the interaction between tumor and stroma required for neoplastic cell migration. Similar to $\mathrm{N}$-cadherin, we found foci of vimentin expression in many adenocarcinomas and squamous cell carcinomas. In adenocarcinomas, fibrotic areas presented more positive foci than nonfibrotic areas. Cords of tumoral infiltrating cells were totally positive for vimentin. The finding of vimentin-positive tumoral cells mainly in fibrotic areas is consistent with other reports that correlate tumor fibrosis with EMT. In 
fact, acquisition of a mesenchymal phenotype is associated with expression of mesenchymal markers and with abundant deposition of extracellular matrix. Similar observations were reported in other models, like progressive renal fibrosis, where the presence of vimentin-positive epithelial cells correlated with increased degree of fibrotic tissue. ${ }^{65,66}$ E-cadherin downregulation and decreased or mislocalized $\alpha$ - and $\beta$-catenins represent early events in this model, which are already evident in preneoplastic lesions. The loss of adherens and tight junction components may initiate a cascade of intracellular signals leading to N-cadherin upregulation and coexpression of mesenchymal markers. This phenotypic switch that represents the hallmark of EMT was only observed in tumors, in late stages of multistep carcinogenesis (Figure 8).

Since the EMT is a common signature to many types of cancer, the signals driving this key event are currently under investigation. ${ }^{67,68} \mathrm{~A}$ number of studies have shown that EMT occurs in response to growth factors. ${ }^{69-73}$ The role of growth factors and cytokines in the pathogenesis of silica-induced lung carcinogenesis has been the object of earlier studies. ${ }^{20-22,74-77}$ It was shown that hyperplastic type II cells present in this model are sites of active production and secretion of TGF- $\beta 1 .^{21,76}$ In rat alveolar cells in culture, silica treatment induced a dramatic secretion of TGF- $\beta 1 .^{77}$ These data raise the possibility that TGF- $\beta$, and other growth factors secreted by the preneoplastic and neoplastic cells, could play a role in inducing the EMT observed in our model.

In conclusion, we have shown that an animal model for multistep lung carcinogenesis mimics the EMT observed in clinical samples and in vitro models. This animal model represents a useful tool

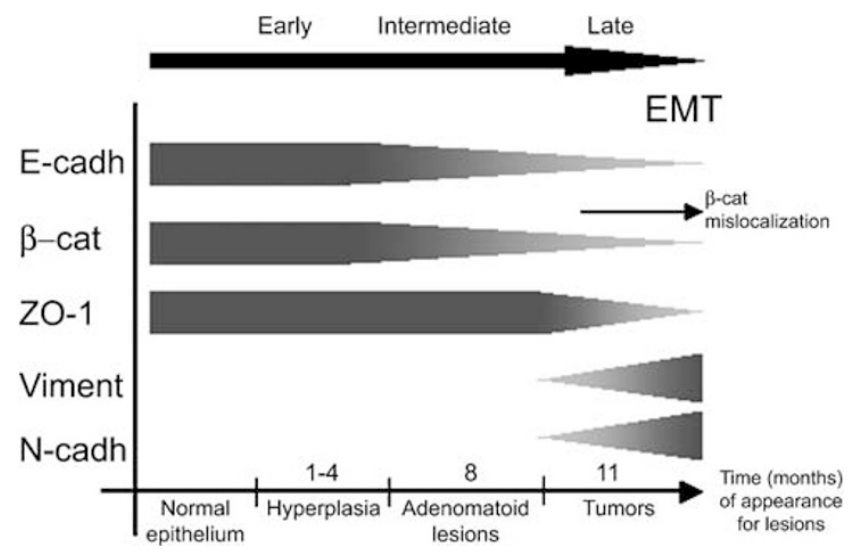

Figure 8 Diagram to illustrate the changes with time of the EMTrelated protein expression in the silica-induced rat lung carcinogenesis model. E-cadherin and $\beta$-catenin downregulation represents an early event that is already evident in preneoplastic lesions. $\beta$-catenin mislocalization was only observed in advanced lesions. Decreased ZO-1 and neoexpression of vimentin and Ncadherin were later events only observed in tumors. This phenotypic switch represents the hallmark of the EMT process observed in this model. to assess the expression of other components involved in this transition and to understand the molecular events driving this process. Future studies, through the systematic analysis of differentially expressed genes, will address the genetic and epigenetic regulation of gene expression and the cell-to-cell and cell-matrix interactions involved in the EMT.

\section{Acknowledgements}

We thank Teresa Sabata and Paz Zamora for technical assistance. This work was supported by the Spanish Ministry of Health/Fondo de Investigaciones Sanitarias (RTIC C03/10), and through the agreement between FIMA and the 'UTE project CIMA'. DB is supported by Gobierno de Navarra scholarship. EE has a grant form the Spanish Ministry of Education and Culture. FL is an investigator of the Ramon y Cajal program. FL is supported by funds from the Ministry of Science and Technology (Plan Nacional I+D+I, Ref. SAF2001-1220), and Government of Navarra (Ortiz de Landázuri Scholarship). US is scientist emeritus at Center for Cancer Research, National Cancer Institute.

\section{References}

1 Knust E, Bossinger O. Composition and formation of intercellular junctions in epithelial cells. Science 2002;298:1955-1959.

2 Tsukita S, Furuse M, Itoh M. Multifunctional strands in tight junctions. Nat Rev Mol Cell Biol 2001;2: 285-293.

3 Jamora C, Fuchs E. Intercellular adhesion, signalling and the cytoskeleton. Nat Cell Biol 2002;4:101-108.

4 Bremnes RM, Veve R, Hirsch FR, et al. The E-cadherin cell-cell adhesion complex and lung cancer invasion, metastasis, and prognosis. Lung Cancer 2002;36: 115-124.

5 Conacci-Sorrell M, Zhurinsky J, Ben-Ze'ev A. The cadherin-catenin adhesion system in signaling and cancer. J Clin Invest 2002;109:987-991.

6 Hirohashi S. Inactivation of the E-cadherin-mediated cell adhesion system in human cancers. Am J Pathol 1998;153:333-339.

7 Esteller M. CpG island hypermethylation and tumor suppressor genes: a booming present, a brighter future. Oncogene 2002;21:5427-5440.

8 Islam S, Carey TE, Wolf GT, et al. Expression of Ncadherin by human squamous carcinoma cells induces a scattered fibroblastic phenotype with disrupted cellcell adhesion. J Cell Biol 1996;135:1643-1654.

9 Tomita K, van Bokhoven A, van Leenders GJ, et al. Cadherin switching in human prostate cancer progression. Cancer Res 2000;60:3650-3654.

10 Thiery JP. Epithelial-mesenchymal transitions in tumour progression. Nat Rev Cancer 2002;2:442-454.

11 Arias AM. Epithelial-mesenchymal interactions in cancer and development. Cell 2001;105:425-431. 
12 Cheng SL, Lecanda F, Davidson MK, et al. Human osteoblasts express a repertoire of cadherins, which are critical for BMP-2-induced osteogenic differentiation. J Bone Miner Res 1998;13:633-644.

13 Prasad S, Soldatenkov VA, Srinivasarao G, et al. Intermediate filament proteins during carcinogenesis and apoptosis (Review). Int J Oncol 1999;14:563-570.

14 Nagle RB. Intermediate filament expression in prostate cancer. Cancer Metast Rev 1996;15:473-482.

15 Ruoslahti E. Fibronectin and its integrin receptors in cancer. Adv Cancer Res 1999;76:1-20.

16 Stewart M. Intermediate filament structure and assembly. Curr Opin Cell Biol 1993;5:3-11.

17 Gilles C, Polette M, Piette J, et al. Vimentin expression in cervical carcinomas: association with invasive and migratory potential. J Pathol 1996;180:175-180.

18 Hendrix MJ, Seftor EA, Seftor RE, et al. Experimental co-expression of vimentin and keratin intermediate filaments in human breast cancer cells results in phenotypic interconversion and increased invasive behavior. Am J Pathol 1997;150:483-495.

19 Mao L. Molecular abnormalities in lung carcinogenesis and their potential clinical implications. Lung Cancer 2001;34(Suppl 2):S27-S34.

20 Saffiotti U, Williams A, Daniel L, et al. Carcinogenesis by crystalline silica: animal, cellular and molecular sudies. In: Castranova V, Vallyathan V, Wallace W (eds). Silica and Silica-induced Lung Diseases. CRC Press: Boca Raton, FL, 1996, pp 345-381.

21 Williams AO, Flanders KC, Saffiotti U. Immunohistochemical localization of transforming growth factorbeta 1 in rats with experimental silicosis, alveolar type II hyperplasia, and lung cancer. Am J Pathol 1993; 142:1831-1840.

22 Saffiotti U, Daniel LN, Mao Y, et al. Biological studies on the carcinogenic mechanisms of quartz. Rev Mineral 1993;28:523-544.

23 Warheit DB. Inhaled amorphous silica particulates: what do we know about their toxicological profiles? J Environ Pathol Toxicol Oncol 2001;20(Suppl 1): 133-141.

24 Brabletz T, Herrmann K, Jung A, et al. Expression of nuclear beta-catenin and $\mathrm{c}-\mathrm{myc}$ is correlated with tumor size but not with proliferative activity of colorectal adenomas. Am J Pathol 2000;156:865-870.

25 Fraga MF, Uriol E, Borja Diego L, et al. Highperformance capillary electrophoretic method for the quantification of 5-methyl 2'-deoxycytidine in genomic DNA: application to plant, animal and human cancer tissues. Electrophoresis 2002;23:1677-1681.

26 Esteller M, Fraga MF, Guo M, et al. DNA methylation patterns in hereditary human cancers mimic sporadic tumorigenesis. Hum Mol Genet 2001;10:3001-3007.

27 Herman JG, Graff JR, Myohanen S, et al. Methylationspecific PCR: a novel PCR assay for methylation status of CpG islands. Proc Natl Acad Sci USA 1996;93: 9821-9826.

28 Esteller M, Sparks A, Toyota M, et al. Analysis of adenomatous polyposis coli promoter hypermethylation in human cancer. Cancer Res 2000;60: 4366-4371.

29 Emmendoerffer A, Hecht M, Boeker T, et al. Role of inflammation in chemical-induced lung cancer. Toxicol Lett 2000;112-113:185-191.

30 Shacter E, Weitzman SA. Chronic inflammation and cancer. Oncology (Huntingt) 2002;16:217-226. 229; discussion 230-232.
31 Ballaz S, Mulshine JL. The potential contributions of chronic inflammation to lung carcinogenesis. Clin Lung Cancer 2003;5:46-62.

32 Bissell MJ, Radisky D. Putting tumours in context. Nat Rev Cancer 2001;1:46-54.

33 Pirinen RT, Hirvikoski P, Johansson RT, et al. Reduced expression of alpha-catenin, beta-catenin, and gammacatenin is associated with high cell proliferative activity and poor differentiation in non-small cell lung cancer. J Clin Pathol 2001;54:391-395.

34 Kase S, Sugio K, Yamazaki K, et al. Expression of Ecadherin and beta-catenin in human non-small cell lung cancer and the clinical significance. Clin Cancer Res 2000;6:4789-4796.

35 Kimura K, Endo Y, Yonemura Y, et al. Clinical significance of S100A4 and E-cadherin-related adhesion molecules in non-small cell lung cancer. Int J Oncol 2000;16:1125-1131.

36 Hao XP, Pretlow TG, Rao JS, et al. Beta-catenin expression is altered in human colonic aberrant crypt foci. Cancer Res 2001;61:8085-8088.

37 Suzuki T, Yano H, Nakashima Y, et al. Beta-catenin expression in hepatocellular carcinoma: a possible participation of beta-catenin in the dedifferentiation process. J Gastroenterol Hepatol 2002;17:994-1000.

38 Huiping C, Kristjansdottir S, Jonasson JG, et al. Alterations of E-cadherin and beta-catenin in gastric cancer. Bmc Cancer 2001;1:16.

39 Tanaka Y, Kato K, Notohara K, et al. Frequent betacatenin mutation and cytoplasmic/nuclear accumulation in pancreatic solid-pseudopapillary neoplasm. Cancer Res 2001;61:8401-8404.

40 Haydon RC, Deyrup A, Ishikawa A, et al. Cytoplasmic and/or nuclear accumulation of the beta-catenin protein is a frequent event in human osteosarcoma. Int J Cancer 2002;102:338-342.

41 Ishigaki K, Namba H, Nakashima M, et al. Aberrant localization of beta-catenin correlates with overexpression of its target gene in human papillary thyroid cancer. J Clin Endocrinol Metab 2002;87:3433-3440.

42 Shibanuma H, Hirano T, Tsuji K, et al. Influence of Ecadherin dysfunction upon local invasion and metastasis in non-small cell lung cancer. Lung Cancer 1998;22:85-95.

43 Rodriguez-Salas N, Palacios J, de Castro J, et al. Betacatenin expression pattern in small cell lung cancer: correlation with clinical and evolutive features. Histol Histopathol 2001;16:353-358.

44 Retera JM, Leers MP, Sulzer MA, et al. The expression of beta-catenin in non-small-cell lung cancer: a clinicopathological study. J Clin Pathol 1998;51: 891-894.

45 Nawrocki B, Polette M, Van Hengel J, et al. Cytoplasmic redistribution of E-cadherin-catenin adhesion complex is associated with down-regulated tyrosine phosphorylation of E-cadherin in human bronchopulmonary carcinomas. Am J Pathol 1998;153:1521-1530.

46 Clavel CE, Nollet F, Berx G, et al. Expression of the Ecadherin-catenin complex in lung neuroendocrine tumours. J Pathol 2001;194:20-26.

47 Hommura F, Furuuchi K, Yamazaki K, et al. Increased expression of beta-catenin predicts better prognosis in nonsmall cell lung carcinomas. Cancer 2002;94: $752-758$

48 Kotsinas A, Evangelou K, Zacharatos P, et al. Proliferation, but not apoptosis, is associated with distinct betacatenin expression patterns in non-small-cell lung 
carcinomas: relationship with adenomatous polyposis coli and G(1)-to S-phase cell-cycle regulators. Am J Pathol 2002;161:1619-1634.

49 Hajra KM, Fearon ER. Cadherin and catenin alterations in human cancer. Genes Chromosomes Cancer 2002; 34:255-268.

50 Kallakury BV, Sheehan CE, Winn-Deen E, et al. Decreased expression of catenins (alpha and beta), p120 CTN, and E-cadherin cell adhesion proteins and E-cadherin gene promoter methylation in prostatic adenocarcinomas. Cancer 2001;92:2786-2795.

51 Yoshiura K, Kanai Y, Ochiai A, et al. Silencing of the Ecadherin invasion-suppressor gene by $\mathrm{CpG}$ methylation in human carcinomas. Proc Natl Acad Sci USA 1995;92:7416-7419.

52 Rodrigo I, Cato AC, Cano A. Regulation of E-cadherin gene expression during tumor progression: the role of a new Ets-binding site and the E-pal element. Exp Cell Res 1999;248:358-371.

53 Bolos V, Peinado H, Perez-Moreno MA, et al. The transcription factor Slug represses E-cadherin expression and induces epithelial to mesenchymal transitions: a comparison with Snail and E47 repressors. J Cell Sci 2003;116:499-511.

54 Cano A, Perez-Moreno MA, Rodrigo I, et al. The transcription factor snail controls epithelial-mesenchymal transitions by repressing E-cadherin expression. Nat Cell Biol 2000;2:76-83.

55 Guaita S, Puig I, Franci C, et al. Snail induction of epithelial to mesenchymal transition in tumor cells is accompanied by MUC1 repression and ZEB1 expression. J Biol Chem 2002;277:39209-39216.

56 Reichert M, Muller T, Hunziker W. The PDZ domains of zonula occludens-1 induce an epithelial to mesenchymal transition of Madin-Darby canine kidney I cells. Evidence for a role of beta-catenin/Tcf/Lef signaling. J Biol Chem 2000;275:9492-9500.

57 Balda MS, Garrett MD, Matter K. The ZO-1-associated Y-box factor ZONAB regulates epithelial cell proliferation and cell density. J Cell Biol 2003;160:423-432.

58 Hoover KB, Liao SY, Bryant PJ. Loss of the tight junction MAGUK ZO-1 in breast cancer: relationship to glandular differentiation and loss of heterozygosity. Am J Pathol 1998;153:1767-1773.

59 Ando-Akatsuka Y, Yonemura S, Itoh M, et al. Differential behavior of E-cadherin and occludin in their colocalization with ZO-1 during the establishment of epithelial cell polarity. J Cell Physiol 1999;179:115-125.

60 Itoh M, Nagafuchi A, Moroi S, et al. Involvement of ZO-1 in cadherin-based cell adhesion through its direct binding to alpha catenin and actin filaments. J Cell Biol 1997;138:181-192.

61 Balda MS, Matter K. Tight junctions. J Cell Sci 1998; 111:541-547.

62 Kim JB, Islam S, Kim YJ, et al. N-cadherin extracellular repeat 4 mediates epithelial to mesenchymal transition and increased motility. J Cell Biol 2000;151:1193-1206.

63 Nieman MT, Prudoff RS, Johnson KR, et al. N-cadherin promotes motility in human breast cancer cells regardless of their E-cadherin expression. J Cell Biol 1999;147:631-644.

64 Hazan RB, Kang L, Whooley BP, et al. N-cadherin promotes adhesion between invasive breast cancer cells and the stroma. Cell Adhes Commun 1997;4: 399-411.

65 Nakatsuji S, Yamate J, Sakuma S. Relationship between vimentin expressing renal tubules and interstitial fibrosis in chronic progressive nephropathy in aged rats. Virchows Arch 1998;433:359-367.

66 Okada H, Ban S, Nagao S, et al. Progressive renal fibrosis in murine polycystic kidney disease: an immunohistochemical observation. Kidney Int 2000;58: 587-597.

67 Boyer B, Valles AM, Edme N. Induction and regulation of epithelial-mesenchymal transitions. Biochem Pharmacol 2000;60:1091-1099.

68 Savagner P. Leaving the neighborhood: molecular mechanisms involved during epithelial-mesenchymal transition. Bioessays 2001;23:912-923.

69 Bhowmick NA, Ghiassi M, Bakin A, et al. Transforming growth factor-beta1 mediates epithelial to mesenchymal transdifferentiation through a RhoAdependent mechanism. Mol Biol Cell 2001;12:27-36.

70 Grande M, Franzen A, Karlsson JO, et al. Transforming growth factor-beta and epidermal growth factor synergistically stimulate epithelial to mesenchymal transition (EMT) through a MEK-dependent mechanism in primary cultured pig thyrocytes. J Cell Sci 2002;115: 4227-4236.

71 Cui W, Fowlis DJ, Bryson S, et al. TGFbeta1 inhibits the formation of benign skin tumors, but enhances progression to invasive spindle carcinomas in transgenic mice. Cell 1996;86:531-542.

72 Yi JY, Hur KC, Lee E, et al. TGFbeta1 -mediated epithelial to mesenchymal transition is accompanied by invasion in the SiHa cell line. Eur J Cell Biol 2002; 81:457-468.

73 DeLuca SM, Gerhart J, Cochran E, et al. Hepatocyte growth factor/scatter factor promotes a switch from Eto N-cadherin in chick embryo epiblast cells. Exp Cell Res 1999;251:3-15.

74 Elizegi E, Pino I, Vicent S, et al. Hyperplasia of alveolar neuroendocrine cells in rat lung carcinogenesis by silica with selective expression of proadrenomedullinderived peptides and amidating enzymes. Lab Invest 2001;81:1627-1638.

75 Saffiotti U. The pathology induced by silica in relation to fibrogenesis and carcinogenesis. In: Goldsmith DF, Winn DM, Shy CM (eds). Silica, Silicosis and Cancer. Praeger: New York, 1986, pp 287-307.

76 Williams AO, Saffiotti U. Transforming growth factor beta1, ras and p53 in silica-induced fibrogenesis and carcinogenesis. Scand J Work Environ Health 1995; 21(Suppl 2):30-34.

77 Williams AO, Knapton AD, Ifon ET, et al. Transforming growth factor beta expression and transformation of rat lung epithelial cells by crystalline silica (quartz). Int J Cancer 1996;65:639-649. 